\title{
Après la leçon de Christophe Cauquil
}

\section{Guy Dufau, Hervé Thiery, Colette Vitse et François Durand-Dastès}

\section{(2) OpenEdition}

\section{Journals}

\section{Édition électronique}

URL : http://journals.openedition.org/trema/2275

DOI : 10.4000/trema.2275

ISSN : 2107-0997

\section{Éditeur}

Faculté d'Éducation de l'université de Montpellier

\section{Édition imprimée}

Date de publication : 1 mai 1994

Pagination : 33-44

ISSN : 1167-315X

\section{Référence électronique}

Guy Dufau, Hervé Thiery, Colette Vitse et François Durand-Dastès, « Après la leçon de Christophe Cauquil », Tréma [En ligne], 5 | 1994, mis en ligne le 01 mai 1994, consulté le 19 avril 2019. URL : http:// journals.openedition.org/trema/2275; DOI : 10.4000/trema.2275

Ce document a été généré automatiquement le 19 avril 2019.

Trema 


\title{
Après la leçon de Christophe Cauquil
}

\author{
Guy Dufau, Hervé Thiery, Colette Vitse et François Durand-Dastès
}

\section{Guy Dufau}

1 Si j'étais dans l'exercice normal de mon métier (ce qui n'est, et de très loin pas le cas) je dirais... Avant de dire je voudrais remercier chaudement Christophe Cauquil d'avoir accepté de se prêter à cet exercice qui ne correspond pas tout à fait à ce qu'il fait habituellement. J'aurais donc dit :

- On est ici dans le droit fil des programmes. Au CM2 (Cours Moyen deuxième année) on aborde l'étude de la région où l'élève vit et sa place dans la France.

- On est ici dans le droit fil des Instructions Officielles. Elles recommandent de donner un petit nombre de connaissances claires et précises, « donner un sens plus clair aux mots de la tribu » disait tout à l'heure R. Brunet. Il convient de soutenir, d'alimenter la conscience nationale - c'est le civisme dont a parlé Monsieur le Recteur. Autrefois on parlait aussi de « commune culture ». C'est vrai depuis « Le tour de la France par deux enfants ». Elles demandent que l'on fournisse à l'élève des repères : à l'histoire de fournir des repères dans le temps et à la géographie de les fournir dans l'espace. Elles prescrivent enfin de donner l'habitude de la rigueur et demandent que l'on aborde, modestement, l'explication ou l'interprétation (avec méthode) de l'organisation de l'espace.

2 Je crois, quant à moi, que tout cela fut fait. Et il fallait le faire, comme on dit.

3 Sur la manière maintenant, il y a peu de remarques à faire :

- C'est le zoom avant qui a été choisi, alors qu'on aurait pu choisir le zoom arrière, cela n'a pas grande importance. J'appelle zoom ce que vous appelleriez peut-être le changement d'échelle. Il fallait répondre à la question Où ? et la démarche choisie a permis de répondre.

- A l'école élémentaire il convient de mettre en ordre «le déjà-là ", comme disent si joliment les pédagogues lorsqu'ils souhaitent parler du savoir, un peu nébuleux parfois, dont les élèves sont porteurs. Cela aussi fut fait. Le récit, les textes sont, avec l'interrogation, les techniques employées pour faciliter l'expression du « déjà-là ». 
4 Je ne parlerai pas des contenus qui sont passés. Tout le monde a vu l'exercice. Je veux insister, pourtant sur les idées « grosses » de développements futurs :

- Il y avait le « patois » (le volem viure al païs)

- La mer, pas loin, et la Tunisie (en face)

- Ils ont pressenti l'écartèlement de la région.

Dans les premiers manuels illustrés, le maintien de l'antique formule catéchistique n'excluait pas la présence d'une taxinomie très rationnelle.

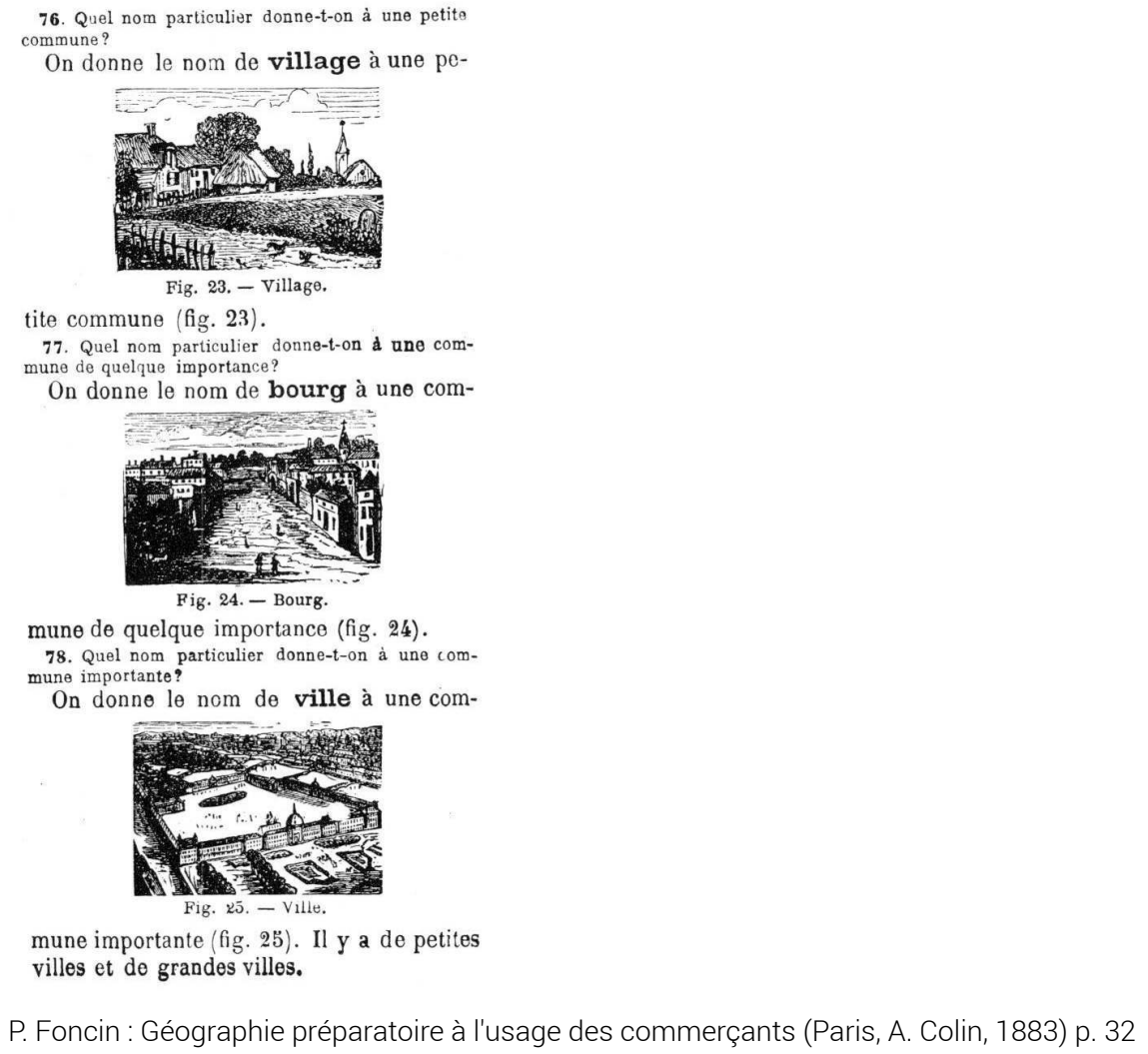

mune importante (fig. 25). Il y a de petites villes et de grandes villes.

P. Foncin : Géographie préparatoire à l'usage des commerçants (Paris, A. Colin, 1883) p. 32

\section{Hervé Thery}

5 Je voudrais voir en quoi cette leçon correspond à l'idée que nous nous faisons (au GIP RECLUS) de la géographie et en quoi cela prépare (c'est ambitieux) à l'évaluation prospective des territoires.

Première question que posent les enfants: qu'est-ce que le métier de géographe? Réponse possible : elle passe par le territoire et ce qu'il est. De la même façon les cabinets ministériels posent la question: que peut-on faire de ce territoire ? La géographie sert aussi et principalement à cette question.

\section{Cinq points}

1. Quand j'évalue un territoire, ce qui n'est pas sans parenté avec ce que fait l'instituteur dans sa classe, je nomme, je situe, je mets en rapport, par rapport au sud : un Midi de la France, sur l'arc méditerranéen. Le Languedoc-Roussillon, comme les autres espaces participe d'une intersection plus large, France, Europe, Méditerranée, etc. 
2. C'est une région, mais aussi des gens : les acteurs. Trop de photos sont vides, ce qui renvoie à la boutade d'origine, l'histoire c'est les gens, la géographie c'est des cartes. Ce qui est faux, on parlera de toutes les populations.

3. Où sont les fractures? Cette région est extraordinairement fracturée, son nom est double, les oppositions nombreuses entre est et ouest, littoral et montagne.

4. Que se passera-t-il après? Qu'arrivera-t-il aux paysages ? L'afflux démographique va-t-il se poursuivre? C'est un record des régions d'accueil, après la région parisienne. S'il en arrive 300000 il faut 150000 emplois rien que pour maintenir ce qui existe. Il ne faut pas hésiter à faire un peu de prospective.

5. En dehors des instructions, à quoi bon parler du Languedoc-Roussillon? Quels espaces d'appartenance? Les enfants se sentent certainement plus de Castelnau, ou plus de la France. Ou de l'Europe? Quels sont les emboîtements d'échelles? Non pas au sens de poupées russes, mais au sens des appartenances multiples et croisées des différentes échelles.

7 On doit pouvoir le dire simplement à des enfants. Ce qui débouche sur des comparaisons intéressantes, pour aborder les lois de l'espace : Où suis-je ? Dans quoi ? Ce sont des voies simples, conformes aux instructions.

Une vieille division héritée de Buache : les bassins hydrologiques.

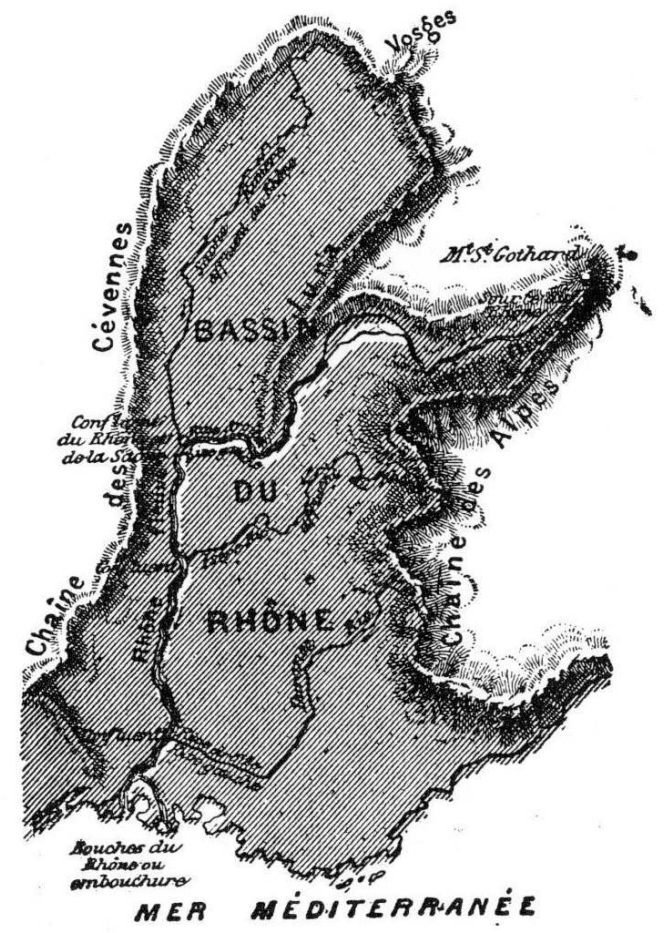

F.ğ. 22. - Bassin du Rhône.

P. Foncin : Géographie préparatoire à l'usage des commerçants (Paris, A. Colin, 1883) p.32

\section{Colette Vitse}

8 Tout comme l'a fait Christophe Cauquil, ce qui suit, en forme de notes cursives, sert de charpente à l'exposé. 
9 Matériel : un rétroprojecteur - dossier de cartes pour les élèves. Nous commençons aujourd'hui l'étude de la région Languedoc-Roussillon... pas de titre pour le moment. Cela ne nous empêche pas de prendre des notes.

10 - Tout de suite prenez la carte $\mathrm{n}^{\circ} \mathrm{l}$ (carte au rétroprojecteur).

un élève présente la carte.

France par départements.

On veut montrer l'accroissement moyen annuel entre 1982 et 1990 avec un regroupement des taux d'accroissement en trois classes.

11 - En vous aidant de la carte $n^{\circ} 2$ localisez le Languedoc-Roussillon sur la carte $n^{\circ} l$, délimitez-le (un élève vient le faire au rétroprojecteur).

Comment pourrait-on situer le Languedoc-Roussillon par rapport aux pays méditerranéens les plus proches?

un élève : au Sud Est l'Italie, au Sud-Ouest l'Espagne.

12 On a pu dire que le Languedoc-Roussillon faisait partie du boulevard de la Méditerranée entre l'Espagne et l'Italie.

Comment pourrait-on le situer dans la France du Sud?

un élève : entre la Provence et le Bassin aquitain.

13 On peut dire que le Languedoc-Roussillon est un « midi médian » entre le midi provençal et le midi aquitain.

14 Sur la carte $n^{\circ} \mathrm{l}$ et par rapport à l'ensemble de la France le Languedoc-Roussillon est-il bien ou mal placé au point de vue de l'accroissement de la population (regardez bien la légende).

un élève : oui, il est parmi les régions de plus fort accroissement.

15 Sur le plan démographique (comme sur le plan économique) quel adjectif peut-on employer pour parler d'une région qui « bouge »?

un élève (mais assez difficilement !) : c'est une région dynamique.

16 Au vue d'une seule carte nous ne pouvons pas affirmer que le Languedoc-Roussillon est dynamique, aussi nous poserons une question et le titre de la leçon sera une interrogation :

17 Le Languedoc-Roussillon, région dynamique?

18 Nous allons essayer, ensemble, de répondre à cette question à travers un certain nombre de cartes portant toutes sur des problèmes humains.

19 - Prenez la carte $n^{\circ} 2$.

un élève présente la carte.

20 Il s'agit cette fois du Languedoc-Roussillon uniquement. Le sujet de la carte est le même que celui de la carte $n^{\circ} 1$.

21 Localisez le nom des différents départements, le nom de la capitale régionale, le nom des préfectures.

(un élève le fait au rétroprojecteur).

22 Analysez la carte maintenant: quelles sont les «ruptures »? (un élève les montre au rétroprojecteur).

Une rupture Nord/Sud (Lozère au Nord, seule).

Une rupture Nord-Est/Sud-Ouest (Gard-Hérault au Nord-Est).

La rupture Nord/Sud est ancienne : elle sépare l'intérieur du littoral plus dynamique. 
La rupture Nord-Est/Sud-Ouest est plus récente : elle coupe le Sud-Ouest plus rural, plus traditionnel qui était le «beau Languedoc » du XIXème siècle (le phylloxéra avait touché l'Est) et le Nord-Est, plus ouvert, plus dynamique, qui est devenu le « beau Languedoc » du XXème siècle.

Explications :

Pourquoi?

Globalement le Languedoc-Roussillon est-il bien placé ? dynamique?

Accroissement moyen annuel pour la France 0,45\%.

D'après cette carte ai-je le droit de dire : le Languedoc-Roussillon région dynamique?

un élève : oui, parce que quatre régions sont situées au dessus de la moyenne française.

Quelles sont les composantes de l'accroissement de la population?

un élève : l'accroissement naturel et le solde migratoire.

- Prenez la carte $n^{\circ} 3$.

Un élève présente la carte.

c'est une carte du Languedoc-Roussillon par départements. On a représenté l'excédent naturel (moyenne annuelle 82-90).

Quels éléments sont pris en compte pour le calcul de l'excédent naturel ?

un élève : les naissances et les décès.

Analysez la carte maintenant : quels sont les différents comportements démographiques? un élève : deux départements (Gard-Hérault) ont un accroissement positif.

Trois départements (Lozère-Aude-Pyrénées Orientales) ont un »accroissement » négatif.

Qu'est-ce que cela signifie?

La mortalité est supérieure à la natalité.

Le Gard et l'Hérault ont une natalité plus forte. Pourquoi?

un élève : population plus jeune et pourquoi?

un autre élève : il y a plus d'emplois.

Dans les trois autres départements c'est la mortalité qui est plus forte.

un élève : la population est plus vieille.

En effet, en Lozère et dans l'Aude il y a plus de difficultés économiques et exode des jeunes; dans les Pyrénées-Orientales plus de personnes âgées (retour au pays après la retraite); de plus dans toute la région, il y a proportionnellement beaucoup de personnes âgées attirées par le climat (héliotropisme).

Recherchez les ruptures comme sur la carte $\mathrm{n}^{\circ} 2$.

La région est-elle dynamique ou pas?

un élève : plutôt non.

Non, la situation démographique naturelle n'est pas très saine.

- Prenez maintenant la carte $\mathrm{n}^{\circ} 4$.

Un élève présente la carte.

c'est une carte du Languedoc-Roussillon par départements.

c'est le solde migratoire annuel qui est représenté.

Quels éléments sont pris en compte dans le calcul du solde migratoire. un élève : les immigrés et les émigrés.

Attention : ici il s'agit des personnes qui entrent et de celles qui sortent de la région Languedoc-Roussillon (sont comptabilisés les Français comme les étrangers). 
D'où viennent les personnes qui entrent dans le Languedoc-Roussillon?

un élève : des personnes venant de l'étranger.

Oui, autrefois les immigrés arrivaient d'Italie et d'Espagne surtout; aujourd'hui ils viennent du Maghreb.

Mais des personnes arrivent d'autres régions: dans le Languedoc-Roussillon les migrations sont surtout interrégionales.

Pourquoi une immigration importante dans le Languedoc-Roussillon?

- plusieurs élèves :

le soleil attire vers le Sud.

les retraités.

il vaut mieux être au soleil quand on est au chômage...

il y a aussi des emplois.

Que savez-vous sur ces créations d'emplois dans le Sud (cours sur les localisations industrielles)?

Synthèse de ce qui s'est dit, la qualité de vie : le Languedoc-Roussillon fait partie de la France des $4 \mathrm{~S}$ (ski-soleil-sable-surf) et c'est ce qui a attiré les emplois liés à la troisième révolution industrielle (emplois du tertiaire qui demandent « plus de matière grise que de matière première »).

1 Quel est le département le plus attractif?

un élève : l'Hérault.

Un nouveau languedocien sur trois s'est établi dans le bassin d'emploi de Montpellier.

Quel est le département le moins attractif?

un élève : la Lozère.

4 Peut-on parler de département « répulsif »?

un élève : non, parce que le solde migratoire est positif.

4 A quoi est dû, globalement, l'accroissement total du Languedoc-Roussillon?

un élève : au solde migratoire surtout.

Oui, à $90 \%$.

Au total la région est-elle globalement dynamique?

un élève : oui.

le dynamisme est-il le même pour tous les départements?

un élève : non

donc le dynamisme est INEGAL.

\section{Conclusion}

47 La leçon devait être traitée en une heure (la deuxième partie et les cartes sur le chômage devaient montrer que le dynamisme était « LIMITE »). Réduite à un peu plus d'une demiheure, le professeur avait prévu, pour ne pas commencer cette deuxième partie sans la terminer, de conclure sur un tableau du « hitparade » des départements qui permettrait de faire la synthèse des trois dernières étudiées.

Sur l'axe accompagnant le tableau la place de chaque département montre bien les trois groupes séparés mais aussi la Lozère... loin derrière... 
Hit parade des départements du Languedoc-Roussillon

\begin{tabular}{|l|c|c|c|c|c|}
\hline \multicolumn{1}{|c|}{ Cartes } & $\mathrm{N}^{\circ} 2$ & $\mathrm{~N}^{\circ} 3$ & $\mathrm{~N}^{\circ} 4$ & Total & Rang \\
\hline Départements & & & & & \\
Aude & 3 & 3 & 3 & 9 & $4 \mathrm{e}$ \\
Gard & 1 & 1 & 3 & 5 & $2 \mathrm{e}$ \\
Hérault & 1 & 1 & 1 & 3 & $1 \mathrm{e}$ \\
Lozère & 5 & 3 & 5 & 13 & $5 \mathrm{e}$ \\
Pyrénées Orientales & 3 & 3 & 2 & 8 & $3 \mathrm{e}$ \\
\hline
\end{tabular}

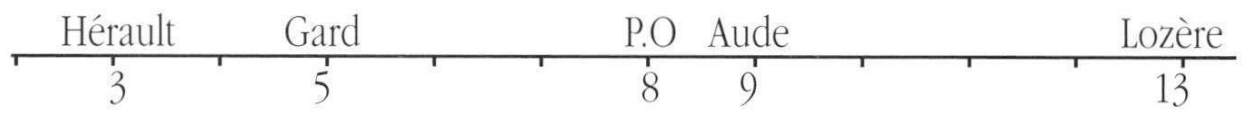

Les premières leçons de géographie faisaient de l'enfant le centre du monde...
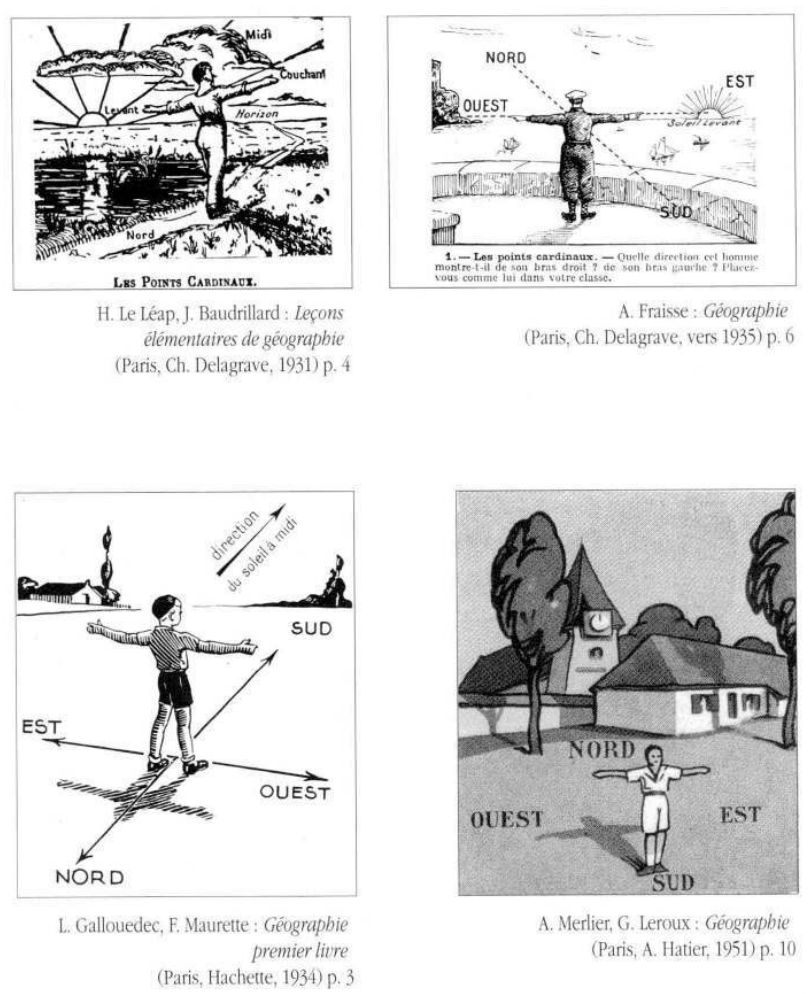

\section{François Durand-Dastes}

Ce qui frappe c'est à quel point les adolescents changent vite lorsqu'ils deviennent adultes, et je souhaiterais dire quelque chose en plus pour lorsqu'ils arriveront à l'université, peut-être pour y faire de la géographie.

Roger Brunet va être content car ce qui m'a frappé, à la fois chez les élèves du CM et chez ceux de Première c'est la façon dont la région Languedoc-Roussillon est une région 
montagne. La plaine, la Méditerranée, la mer, cela nous a été rappelé avec beaucoup d'insistance, sont là, la montagne, au contraire est absente.

La situation de la Lozère le montre, on a assez peu parlé de son relief.

L'éloignement à l'arc méditerranéen est considéré comme beaucoup plus important. Peut-être y a-t-il là quelque chose dont on pourra discuter : le fait de montrer un monde modélisé ne suppose pas qu'on montre pour autant un monde plat parce qu'il ne l'est pas.

Deuxièmement, en jouant un peu sur la langue de bois il est intéressant de voir apparaître en classe de Première des notions qui n'ont pas été nommées mais qui sont présentes. C'est en les nommant dans les premières années de l'enseignement universitaire que je me suis créé la réputation de quelqu'un qui est horriblement abstrait, dans ses enseignements.

D'abord l'utilisation de la technique de classement multivariée, la carte finale, l'échelle finale, c'était bien fait sans le dire. Pourquoi ne pas le dire en Première ou en Terminale, en utilisant la technique des sommes de rangs? On trouvait trois groupes de départements destinés à être repris ultérieurement.

Puis on a vu à un moment donné s'enclencher une boucle de rétroaction, admirable mais pas davantage dite, avec l'idée que quand il y a déficit migratoire, un solde migratoire défavorable, il y a vieillissement de la population, et quand il y a vieillissement de la population, il y a baisse de l'accroissement naturel, et quand il y a baisse de l'accroissement naturel, il y a évidemment baisse de la population... Inconvénient supplémentaire, le vieillissement entraîne aussi l'augmentation de la mortalité. Autre notion qui est apparue, quelque chose de très sain méthodologiquement, la notion de relation de causalité médiate.

L'élève disait un mauvais solde naturel découle du secteur primaire, puis est apparue après l'échange avec le professeur l'idée que parce qu'il y avait du secteur primaire, il y avait moins de dynamisme démographique, donc un solde migratoire négatif, donc un vieillissement de la population, donc un solde naturel négatif.

Très rapidement on a vu ainsi comment l'on passait d'une relation un peu brute à une formulation en termes médiatisés.

Il aurait fallu insister sur ce triomphe. Tout naturellement plus tard ils vont manipuler les relations de causalité ou bien ils ne reconnaîtront pas, lorsque on leur parlera de causalité complexe ou de convergence, les notions qu'on leur aura inculquées. C'est une question à se poser : à quel moment faut-il introduire le nom d'un processus intellectuel que l'on met en place?

Certainement pas au CM, en Première peut-être, mais en premières années de l'université on se heurte à des résistances.

Une dernière chose : il est apparu un très grand contraste entre le groupe Aude-Pyrénées Orientales et Hérault-Gard, il apparaissait qu'il s'agissait d'une inversion, notion très intéressante, et récente. L'explication esquissée n'est qu'un tout début, la grande question -ce qu'à dit Roger Brunet tout à l'heure c'est : il est très important que les élèves gardent en face de la variété, de la réalité du monde, leurs facultés d'étonnement, qu'il ne faut pas fermer par une explication immédiate. Il y a là un phénomène que l'on voit mal, avec seulement quelques pistes, et il faut savoir le dire. Remarque tout à fait injuste puisque toute la leçon n'a pas été faite, qu'elle était prévue pour être poursuivie ultérieurement. Un dernier regret pour un originaire d'un peu à l'Ouest de Toulouse, 
l'accaparement du terme Languedoc par une région autre que celle dont la capitale traditionnelle était tout de même Toulouse.

\section{AUTEURS}

\section{GUY DUFAU}

IPR-IA, responsable de la formation 1er degré à l'IUFM de Montpellier

\section{HERVÉ THIERY}

Directeur du GIP/RECLUS

\section{COLETTE VITSE}

Professeur au lycée Clémenceau de Montpellier

\section{FRANÇOIS DURAND-DASTÈS}

Professeur de géographie à l'université de Paris VII 